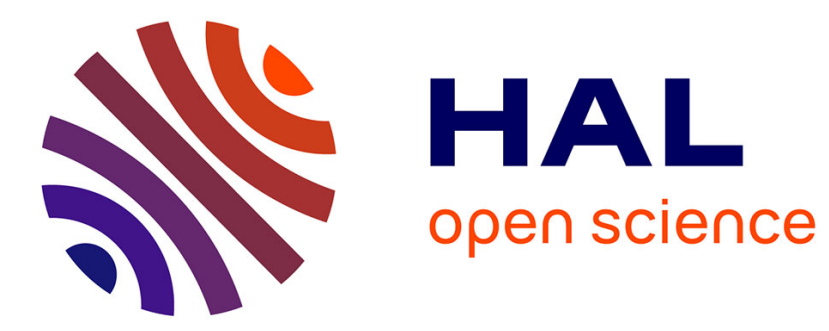

\title{
Tunable light source from large band conversion of continuum in a quadratic crystal
}

F. Baronio, Matteo Conforti, C de Angelis, M Andreana, A. Tonello, V

Couderc

\section{- To cite this version:}

F. Baronio, Matteo Conforti, C de Angelis, M Andreana, A. Tonello, et al.. Tunable light source from large band conversion of continuum in a quadratic crystal. Laser Physics Letters, 2012, 9 (5), pp.359-362. 10.7452/lapl.201110139 . hal-02394943

\section{HAL Id: hal-02394943 \\ https://hal.science/hal-02394943}

Submitted on 5 Dec 2019

HAL is a multi-disciplinary open access archive for the deposit and dissemination of scientific research documents, whether they are published or not. The documents may come from teaching and research institutions in France or abroad, or from public or private research centers.
L'archive ouverte pluridisciplinaire HAL, est destinée au dépôt et à la diffusion de documents scientifiques de niveau recherche, publiés ou non, émanant des établissements d'enseignement et de recherche français ou étrangers, des laboratoires publics ou privés. 
Abstract We consider the conversion of broadband infrared light, generated from photonic crystal fibers, into narrow-band visible pulses, through frequency conversion in quadratic biaxial crystals. We exploit the nonlinear envelope technique to predict cubic and quadratic ultrabroadband dynamics and to guide the experiments. The combination of photonic crystal fibers and quadratic crystals provides the possibility to achieve low-cost laser-light sources with a great flexibility of tuning the spectral properties and covering wavelengths from UV to the infrared range.

Experimental a) and numerical b) spectral traces of visible light generated through photonic crystal fibers and quadratic crystals
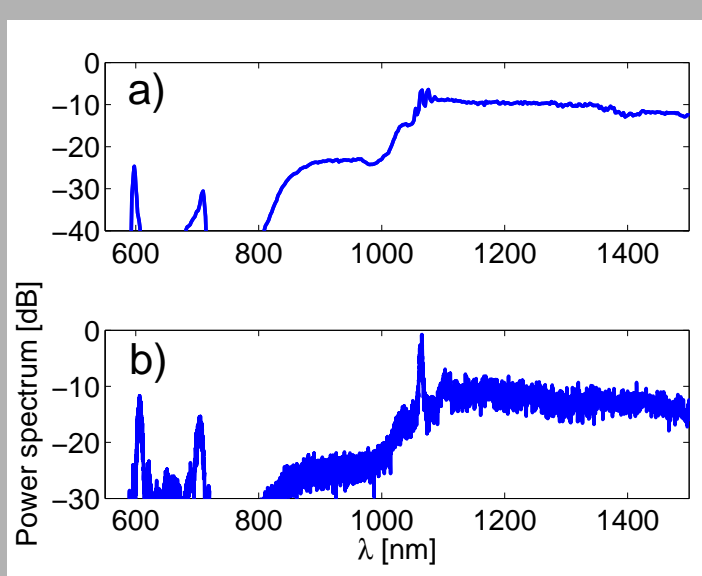

Copyright line will be provided by the publisher

\title{
Tunable light sources from photonic crystal fibers and quadratic crystals
}

\author{
F. Baronio ${ }^{1, *}$, M. Conforti ${ }^{1}$, C. De Angelis ${ }^{1}$, M. Andreana $^{2}$, A. $_{\text {Tonello }}{ }^{2}$, V. Couderc $^{2}$ \\ ${ }^{1}$ Dipartimento di Ingegneria dell'Informazione, University of Brescia, Via Branze 38, Brescia 25123, Italy \\ ${ }^{2}$ XLIM, CNRS and University of Limoges, 123 av. Albert Thomas, Limoges 87060, France.
}

Received: ... .

Published online: ...

Key words: Optical sources and standards; Laser optical system: design and operation; Nonlinear Optics PACS: 42.72.-g, 42.60.-v, 42.65.-k

\section{Introduction}

The availability of light sources with specific and tunable spectral properties is of interest in many fields of physics, from spectroscopy [1,2,3] to spectral imaging [4], from optical sensing to material processing $[5,6]$. Solid state lasers $[7,8,9]$ may offer the chance for tuning the laser wavelength in a limited range. Other more complex lasers, such as dye lasers, can also provide wider wavelength tuning ranges. The generation of complex wavelength distributions, however, would require the superposition of several light sources.

Nowadays, the development of modern supercontinuum (SC) fiber light sources offers novel opportunities for tunable light sources and complex wavelength distributions
$[10,11,12]$. SC light sources are designed to provide a flat spectrum over a wide wavelength range, but limited options for spectral shaping are related to the dependence of the produced spectrum on parameters such as fiber material and structure, fiber length, pump power, pump wavelength. SC light sources are compact and can generate various wavelength distributions with high spatial coherence and high spectral intensity. They are adapted for waveguide coupling and focusing to small spot sizes.

A general and extremely flexible option for shaping the spectrum of such light sources could be provided by combination with quadratic nonlinear crystals [13,14]. Moreover, a comprehensive framework to study the nonlinear evolution of ultrabroadband pulses

\footnotetext{
* Corresponding author: e-mail: baronio@ing.unibs.it
} 
in quadratic nonlinear media has been recently discovered $[15,16]$.

In this Letter, we consider the conversion of broadband infrared light, generated from photonic crystal fibers (PCFs), into narrow-spectrum visible pulses, thorough frequency conversion in quadratic crystals. We exploit the nonlinear envelope technique $[15,16]$ to simulate the cubic and quadratic ultrabroadband dynamics and to guide the experiments. The combination of PCFs and quadratic crystals could provide the possibility to achieve low cost laser-light sources with a great flexibility in tuning the spectral properties and covering wavelengths form UV to the infrared range.

\section{Supercontinuum fiber light source and quadratic crystals}

Supercontinuum light generation has been developed very successfully during recent decades as a powerful concept for wideband fiber light sources [17]. It relies on third order nonlinear effects such as four-wave mixing, solitons and cross-phase modulation. Efficient supercontinuum light generation requires well-defined dispersion conditions. Microstructured PCFs offered new and flexible ways to manipulate the light generation process. Special glasses with high nonlinearity can be used in the fiber core region. By changing the structure of the cladding, the linear and nonlinear properties of the PCF can be adapted for pumping with different sources in order to generate the targeted wavelength ranges. In silica PCFs, numerous studies have demonstrated SC generation covering a wavelength range from UV (around $350 \mathrm{~nm}$ ) to near-IR. By replacing the silica with other glasses such as tellurite or SF6 material, the SC spectrum is shifted towards the higher infrared wavelengths up to about $4 \mu \mathrm{m}$. In spite of the great flexibility in generating such a wide range of wavelengths, it is not already demonstrated the possibility to achieve a specific wavelength spectrum directly by designing the fiber or other system parameters.

A single-pass frequency doubler device may convert the SC broadband infrared radiation into narrow spectrum visible pulses with a tunable carrier wavelength, desired temporal durations, and good-efficiency conversion. We exploited the nonlinear envelope technique to simulate cubic and quadratic ultrabroadband dynamics and to guide the experiments $[15,16]$.

For a demonstration experiment the setup depicted in Fig. 1 is employed. A passively Q-switched Nd:YAG laser delivering $800 \mathrm{ps}$ pulses at $1064 \mathrm{~nm}$ is used as a pump source. The repetition rate and the peak power were $8 \mathrm{kHz}$ and $10 \mathrm{~kW}$, respectively. Two half wave plates and a polarizer allowed to control the power and polarization direction of pump radiation. The pump is coupled into a PCF by an aspheric lens with a coupling efficiency of $75 \%$. For fiber supercontinuum generation, we used a PCF with a multi-component glass structure in the core in order to

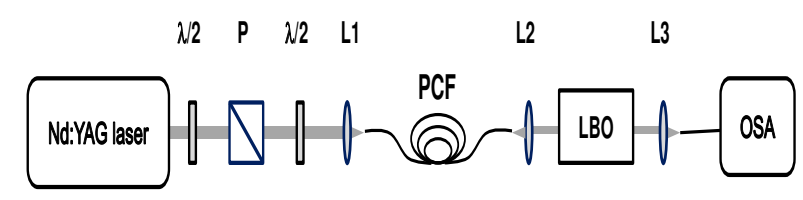

Figure 1 Experimental setup. Nd:YAG laser pump delivering $800 \mathrm{ps}$ pulses, at $1064 \mathrm{~nm}, 8 \mathrm{kHz}$ repetition rate; L1, L2, L3 lenses; $\lambda / 2$ half wave plate; $\mathrm{P}$ polarizer; photonic crystal fiber (PCF); quadratic biaxial LBO crystal; optical spectrum analyzer (OSA).
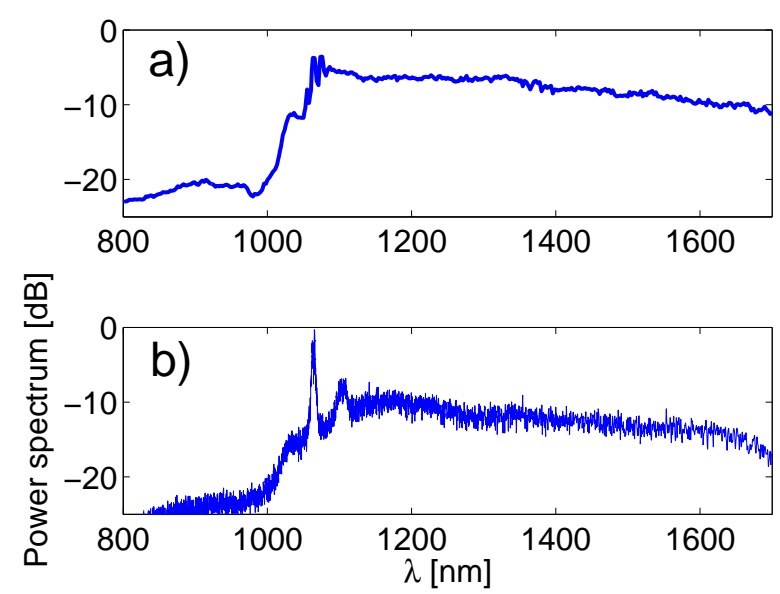

Figure 2 Typical SC spectrum at the output of the PCF. a) OSA meausurement, b) numerical trace.

increase the nonlinearity of the waveguide [18]. By coupling the pump radiation into a $8 m$ long fiber, we generated a broadband spectrum induced by the combination of four-wave mixing, solitonic and cross-phase modulation effects. We considered a compact SC system with a wide wavelength range, which then can be easily manipulated externally. The SC radiation is then focused onto a $2 \mathrm{~cm}$ long quadratic biaxial LBO crystal [19]. As an example, we show the case of light propagation in the principal plane $X Y$ of the biaxial crystal with the azimuthal angle $\phi$ varying from $0^{\circ}$ to $20^{\circ}$. The spectra were subsequently characterized by an optical spectrum analyzer (OSA).

\section{Results and discussion}

Fig. 2a reports a typical experimental SC spectrum at the output of the PCF, characterized by the OSA. Fig. 2b reports the spectrum obtained through standard numerical 

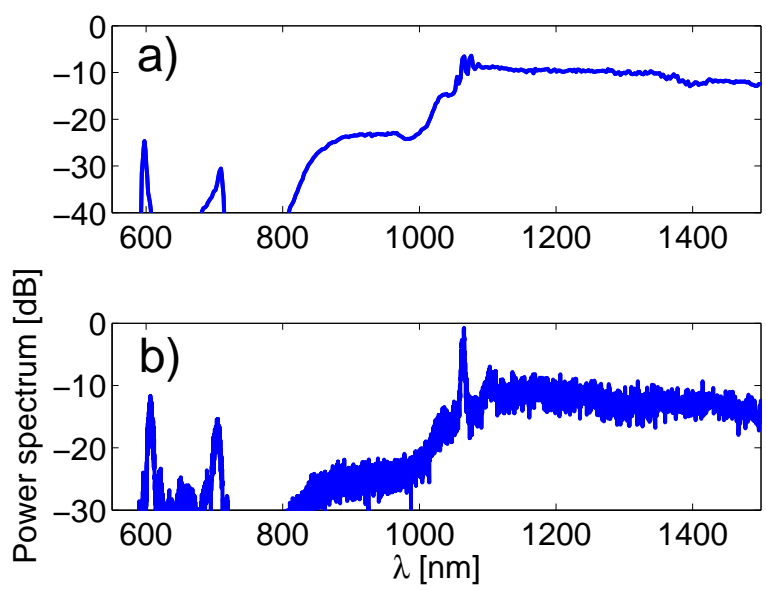

Figure 3 Spectrum at the output of the LBO crystal. a) OSA meausurement, b) numerical trace. The azimuthal angle $\phi=0^{\circ}$.

simulations, averaged over 30 measurements to mimic the integration time of the OSA. The SC extended in the IR range $(1064-2220 \mathrm{~nm})$ for an average power of $50 \mathrm{~mW}$. Fig. 3a reports a typical experimental spectrum at the output of the quadratic LBO crystal. Fig. 3 b reports the spectrum obtained through broadband numerical simulations $[15,16]$. We observed the generation of visible signals at $600 \mathrm{~nm}$ and $700 \mathrm{~nm}$. In fact, second harmonic generation for type I processes takes place in the quadratic crystals [20]. Fig. 4 reports typical temporal profiles of signals generated in the visible range: trains of $200 \mathrm{fs}$ pulses. The use of LBO crystals permits the generation of two visible waves spatially and temporally overlapped, but spectrally separated. The carrier wavelengths of the two waves can be tuned simply varyng the azimuthal angle of the LBO crystal. Fig. 5a reports experimental visible spectra at the output of the quadratic LBO crystal, at different azimuthal crystal angle $\phi$. Fig. 5b shows the contour plots of the normalised parametric gain for second harmonic generation varying the crystal azimuthal angle (the curves are calculated from Sellmeier equations for LBO at temperature $T=27^{\circ}[19]$ ).

The use of the nonlinear envelope technique, thorough reverse engineering [13], would allow to choose different crystals permitting the generation of waves in different regions of the spectrum and/or with desired temporal durations. As an example, the combination of a PCF designed for broadband SC generation in the visible and a KDP crystal would lead to the generation of fields in the UV spectrum.

\section{Conclusion}

In this Letter, we have shown the potential of the nonlinear envelpe technique in describing ultrabroadband

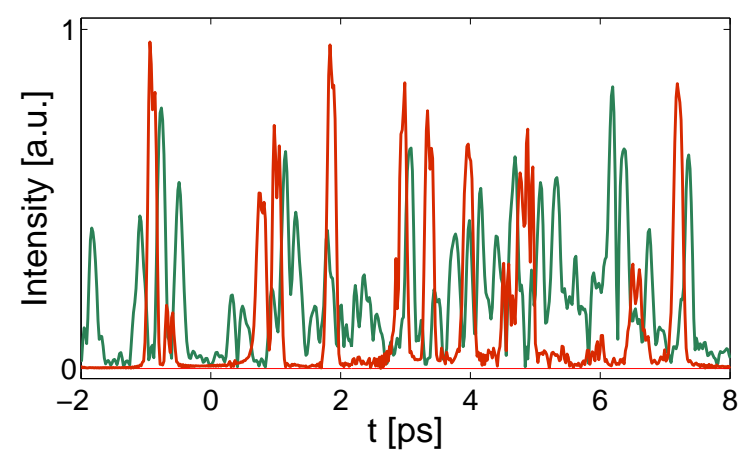

Figure 4 Typical numerical temporal profiles at $600 \mathrm{~nm}$ (green line) and $700 \mathrm{~nm}$ (red line) at the output of the LBO crystal.

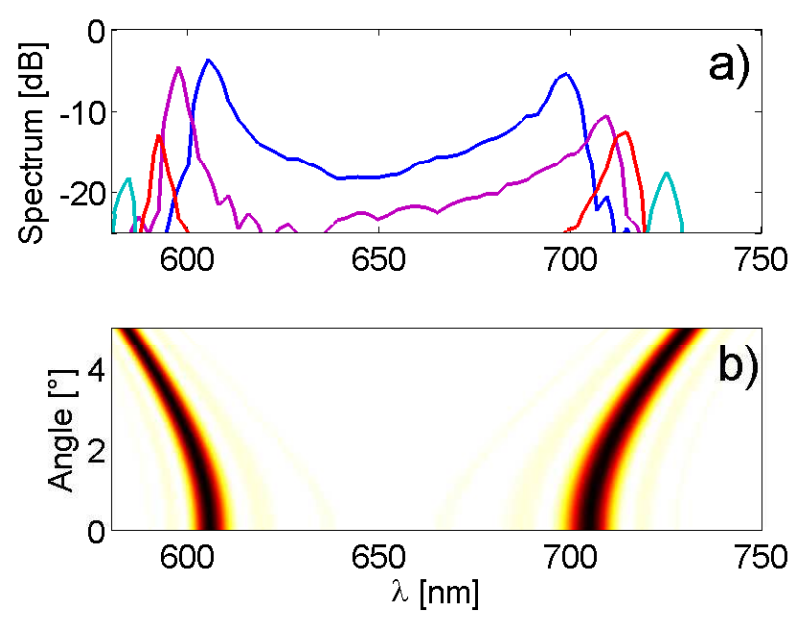

Figure 5 a) OSA meausurement of visible spectra at the output of the LBO crystal at different crystal azimuthal angles. b) Parametric gain for second harmonic generation in LBO as a function of generated wavelength and crystal azimuthal angle.

phenomena. We have considered the conversion of broadband infrared light, generated from a photonic crystal fiber, into narrow spectrum visible pulses, through parametric frequency conversion in a quadratic LBO biaxial crystal. The combination of photonic crystal fibers and quadratic crystals would provide the possibility to achieve low-cost laser-light sources with a great flexibility in shaping the spectral and temporal properties and covering wavelengths form UV to the infrared range. These optical sources could be of interest in spectroscopy, spectral imaging, optical sensing and material processing.

Acknowledgements The present research is supported by PRINMIUR grant $n^{\circ} 2009 P 3 K 72 Z$ and by Fondazione CARIPLO grant $n^{\circ} 2010-0595$. 


\section{References}

[1] E. Vater, G. Bergner, D. Akimov, S. Schlucker, H. Bartelt, B. Dietzek, and J. Popp, Laser Phys. Lett. 6, 639 (2009).

[2] H. Bai, P. Chen, H. Fang, L. Lin, G.Q. Tang, G.G. Mu,W. Gong, Z.P. Liu, H. Wu, H. Zhao, and Z.C. Han, Laser Phys. Lett. 8, 78 (2011).

[3] G. Bergner, D. Akimov, S. Schlucker, H. Baetelt, B. Dietzek, and J. Popp, Laser Phys. Lett. 8, 541 (2011).

[4] X.S. Jiang, S. Chen, J.X. Chen, X.Q. Zhu, L.Q. Zheng, S.M. Zhuo, and D.J. Wang, Laser Phys. 21, 1661 (2011).

[5] P. Chen, Q. Tian, S.J. Baek, X.L. Shang, A. Park, Z.C. Liu, X.Q. Yao, J.Z. Wang, X.H. Wang, Y. Cheng, J. Peng, A.G. Shen, and J.M. Hu, Laser Phys. Lett. 8, 547 (2011).

[6] O. Samek, P. Zemanek, A. Jonas, and H.H. Telle, Laser Phys. Lett. 8, 701 (2011).

[7] Y. Liu, J. Liu, C.C. Liu, R.L. Niu, L.H. Zheng, L.B. Su, and J. Xu, Laser Phys. 21, 472 (2011).

[8] C.L. Li, H.X. Cai, L.J. Xu, Y. Tan, G.Y. Jin, and J. Bi, Laser Phys. 21, 1159 (2011).

[9] M. Andreana, F. Baronio, M. Conforti, A. Tonello, C. De Angelis, and V. Couderc, Laser Phys. Lett. 8, 795 (2011).

[10] V. Tombelaine, G. Bergner, E. Vater, S. Schlucker, B. Dietzek, J. Popp, H. Bartelt, R. Jamier, Opt. Comm. 284, 1970 (2011).

[11] L.R. Wang, X.M. Liu, Y.K. Gong, D. Mao, and L.N. Duan, Laser Phys. 21, 1797 (2011).

[12] G.S. Qin, X. Yan, M. Liao, A. Mori, T. Suzuki, and Y. Ohishi, Laser Phys. 21, 1115 (2011).

[13] M. Conforti, F. Baronio, C. De Angelis, Opt. Lett. 32, 1779 (2007).

[14] M. Marangoni, D. Brida, M. Conforti, A.D. Capobianco, C. Manzoni, F. Baronio, G. Nalesso, C. De Angelis, R. Ramponi, G. Cerullo, Opt. Lett. 34, 241 (2009).

[15] M. Conforti, F. Baronio, C. De Angelis, Phys. Rev. A 81, 053841 (2010).

[16] M. Conforti, F. Baronio, C. De Angelis, IEEE Photonics J. 2, 600 (2010).

[17] J.M. Dudley, G. Genty, S. Coen, Rev. Mod. Phys. 78, 1135 (2006).

[18] V. Tombelaine, A. Labruyere, J. Kobelke, K. Schuster, V. Reichel, P. Leproux, V. Couderc, R. Jamier, H. Bartelt, Opt. Express 17, 15392 (2009).

[19] V.G. Dmitriev, G.G. Gurzadyan, and D.N. Nikogosyan, Handbook of Nonlinear Optical Crystals (Springer, Berlin, 1999).

[20] M. Conforti, F. Baronio, C. De Angelis, J. Opt. Soc. Am. B 28, 1231 (2011). 